\title{
EARTHQUAKE DAMAGE DETECTION USING SATELLITE IMAGES (CASE STUDY: SARPOL-ZAHAB EARTHQUAKE)
}

\author{
Hassan. Aali ${ }^{1}$, Alireza. Sharifi ${ }^{2}, *$, Abbass. Malian ${ }^{2}$ \\ ${ }^{1}$ Graduate Student of Remote Sensing, Shahid Rajaee Teacher Training University,Tehran,Iran \\ ${ }^{2}$ Department of Surveying Engineering Shahid Rajaee Teacher Training University,Tehran,Iran
}

\begin{abstract}
KEY WORDS: Earthquake; Sarpol-Zahab; Remote Sensing; Change Detection;Detection of Changes; Image Classification; Satellite Images
\end{abstract}

\begin{abstract}
:
After the earthquake in Sarpol-e Zahab city, many people were killed or wounded and many buildings were destroyed. After such a destructive event, it is of great interest to efficiently identify the magnitude and the extent of the damaged areas. Remote sensing is an excellent technology for this purpose. Usually, a higher success rate can be achieved when both pre and post-event data, especially multi-view data, are used. Whereas when only post-event data are available, the detection is usually limited to the block level unless VHR images of a resolution of $0.5 \mathrm{~m}$ or higher are involved.The available dataset consists of one Pleiades-1 satellite optical image (post-event), two Sentinel-2 satellite images (pre\&post event).After classification of the sentinel images(pre\&post event) and preparation change maps by means of SVM and the neural network classification methods, two change maps will be provided. Then, A reference change map is prepared with ROIs. For this purpose, on the Pleiades-1 image (after the earthquake), ROIs in two categories "change" and "no change" are defined. In the last step, using the confusion matrix, two change maps from the Sentinel image are compared to the reference image, and the results are analyzed. The producer's accuracy for detecting the collapsed buildings in the SVM classification method was found to be $78.34 \%$ and for the neural network classification was found to be $72.43 \%$. The results show that the change map of the pre- and post-earthquake medium-resolution satellite images such as Sentinel-2 can reveal the collapsed buildings caused by the earthquake successfully.
\end{abstract}

\section{INTRODUCTION}

An earthquake is the most significant natural disaster which destroys millions of properties and kills people due to the collapse of buildings and infrastructures in epicentral and surrounding regions. In this regard, it requires extensive and immediate field investigation to record damage patterns. Investigations of damage levels and their distribution after a destructive earthquake are of primary importance for planning rescue activities at first and for disaster prevention at the second. More than a million earthquakes occur worldwide every year, which equates to roughly two earthquakes per minute. During the years of 2001-2011 only disasters have caused more than 780,000 deaths, with earthquakes accounting for nearly $60 \%$ of all disaster-related mortality [1,2].

Earthquake-induced building damage is one of the most critical threats to cities. The area and the amount of damage, the rate of collapsed buildings, the grade of damage in the affected area, and the type of damage occurred by each building are essential information for successful rescue and reconstruction in disaster areas [3].

Natural disasters such as earthquakes can take thousands of human lives, cause extensive destruction to infrastructure, flatten buildings, and dramatically change the land surface. After occurring a disaster, knowing the location and extent of damaged buildings over a large affected area is vital for emergency actions and rescue works.

Earthquakes, besides leaving psychological effects on human, causes a lot of devastating economic effects. In fact, the earthquake is one of the natural disasters that its destructive effects on the soul of the survivors will remain for many years. And although these people survived in most cases, their house, family, properties, friends, and in the sense of the whole world, all of their financial and emotional resources are lost.

Apart from the emotional and human dimensions of the earthquake, the devastating phenomenon of earthquakes in urban and rural areas has a damaging effect on the economy and totally destroys the economic infrastructure. [4,5,6].

Among other effects of an earthquake are core business activities recession, human and social damage, unemployment, etc., which lead to cause more social and human damages. The number of casualties, injuries, and the disabled are another aspect.

Iran has a specific tectonic position, which has transformed our country into one of the earthquake-stricken parts of the world, and inevitably we experience an average of one or two earthquakes between 6 and 7 magnitudes every year in different parts of the country. [7].

During the past 40 years, there have been 24 major earthquakes of 5.5 magnitudes in Iran, of which, the Manjil, Rudbar, and Bam earthquakes each had the highest human casualties, with more than 25,000 deaths, as well as in 2002 from 170 The million people affected by the earthquake in the world have been responsible for 19 million people. $18 \%$ of the earthquakes have been devastating, and $80 \%$ of them occur among six countries in the world, which Iran is one of them. [7,8].

One of the destructive earthquakes that destroyed urban and rural constructions, completely or parts of them, is the recent earthquake which occulted in Kermanshah, and according to the province's seismographic center, more than 100 aftershocks have been recorded since its inception in Kermanshah.

Statistics show that the highest casualties of an earthquake in Iran are related to Sarpol-e Zahab and Qasr-e Shirin, the reason for the high death toll in these areas was being near the earthquake center. Sarpol-e Zahab is one of the border towns of Kermanshah

\footnotetext{
* Corresponding author-a_sharifi@sru.ac.ir
} 
province in Iran. It is located in the west of the country, and the Zagros Mountains, from the largest mountain range, located in this area. Based on the general population and housing census in 2016, its population was 45481 people (12850 households).

A severe earthquake (magnitude 7.3) on November 12, 2017, shook the provinces of Kermanshah, Ilam and Kurdistan and widespread parts of the west and northwest of the country. Due to its proximity to the city of Sarpol-e Zahab, the intensity of this earthquake caused the greatest damage in the city and left more than 738 dead and many destructed buildings. Sarpol-e Zahab hospital has also been destroyed.

After the earthquake in Sarpol-e Zahab city, many people were killed or wounded and many buildings were destroyed. This severe earthquake caused extensive damages to buildings, facilities, and drastically changed the land surface of the damaged region and a huge number of human, structural, and socioeconomic losses were brought due to it. After such a destructive event, it is of great interest to efficiently identify the magnitude and the extent of the damaged areas. Remote sensing is an excellent technology for this purpose. With a repeated acquisition of the synoptic view images of the earth surface in short time periods, it has become possible to determine the extent and types of changes in the land surface through remote sensing satellites. $[8,9,10]$.

The technology of remote sensing could play an important role in quickly detecting damage distribution before starting the rescue operation, and thus evaluating earthquake effects. Although humans cannot prevent earthquakes, we can change the way we respond to them. Fortunately, remote sensing techniques, both spaceborne and airborne, can make a very effective contribution [11].

The time factor is an important part of the disaster situation and in such a situation, remote sensing can be a great boon. Remote sensing is the science of acquiring, processing and interpreting images that record the interaction between the electromagnetic energy and materials . With the advancement of technology, remote sensing satellites have become a great tool in disaster studies and found wider application including building damage detection [12].

Aerial and space images are excellent data sources that can be used for detecting the post-quake changes. Change detection is a process of identifying differences in the state of a geographical object by observing it at different times [13].

Satellite imagery can be an effective solution to obtain a map of the damaged area, both in supporting the rescue operations and in providing an extensive evaluation of earthquake effects on the built estate.

This assessment can be reinforced by the availability of pre-event images and the application of change detection techniques. Although the reduction of the satellite image pixel size as a result of the availability of VHR satellite data has facilitated the recognition of individual objects such as houses, it poses a challenge to classical pixel-based change detection and image classification methods. [14].

Traditional pixel-based classification approaches have been widely used to characterize and discriminate between different types of image features in both classification and change detection applications involving agricultural, forest, and environmental monitoring.

These methods are based on the concept that semantic information is represented in single pixels and are well suited for non-complex image scenes (e.g., spectrally homogeneous object classes). However, these methods have produced unsatisfying results in the case of applications involving the characterization of man-made structures and other heterogeneous features in urban environments [15].
The main reason is that urban objects are distinguished better through their spatial (i.e., texture, form, area) rather than spectral reflectance properties [16]. In addition, traditional pixel-based classification methods cannot differentiate easily between object features that display high spectral overlap, such as, building roofs from pavements that are constructed using similar material [10]. Furthermore, in applications where VHR satellite imagery is used, it is necessary to expand the object feature base to include spatial characteristics in addition to spectral ones. Objectoriented segmentation and classification approaches offer possibilities to overcome these problems.

These methods take into account knowledge of neighborhood pixels when seeking to determine the most appropriate class for a pixel. They tend to develop a thematic map that is consistent both spectrally and spatially. However, the degree to which adjacent pixels are strongly correlated will depend on the spatial resolution of the satellite sensor and the scale of the image features. For example, adjacent pixels over agricultural land will be strongly correlated, whereas, for the same sensor, adjacent pixels in a busier urban region will not show a strong correlation. Object-oriented methods are generally based on the concept that important semantic information is not represented in single pixels alone but in meaningful image objects and their mutual relations (i.e., context). [18,19].

In this paper, various methods of image classification are presented to compare the results of classification methods such as a supervised non-parametric statistical learning technique or Support vector machines (SVMs) are applied to a high resolution satellite data image and a medium resolution (Sentinel-2) to identify damaged structures. The first purpose of this paper is to obtain an appropriate image of earthquake damage, in order to obtain an appropriate map for it. The second purpose is to examine the various applications of the map of the changes made from the images. With this perfect technology, we can make the world a better places for living.

\section{MATERIALS \& METHOD}

\subsection{Study Area}

The selected site of study is the city of Sarpol-Zahab, one of the areas most strongly hit by the earthquake.

Sarpol- Zahab is one of the counties of Kermanshah province with a total area of $1271 \mathrm{~km}$. This city dates back to 2800 BCE. This border town of Iran was severely damaged during the IranIraq war but was restored after the war. Sarpol-e Zahab is of the ancient borderlands and auriferous regions of Kermanshah province. This county has a very beautiful nature.

The city is $45^{\circ} 52$ 'east longitude, $34^{\circ} 24^{\circ}$ latitude, in the west of the country, and the slopes of the Zagros Mountains are located on the international road of Tehran-Baghdad. Sarpol-Zahab city, from the north, with the city of Sallath-Baba-Jani; from the west to Qasr-e-Shirin city; from the south to the Gilan-e-Gharb, and from the west, along with Iraq and Qasr-e Shirin.

Sarpol-Zahab is a vast plain surrounded by heights and hills. Alvand's permanent river passes through the city and its climate is relatively warm and semi-arid. Based on archeological studies of ancient works and inscriptions, Sarpol-Zahab has had a residence in the Neolithic period (seven thousand years ago). Based on the general census of population and housing in 2016, its population was 45,481 people (12,850 households). 


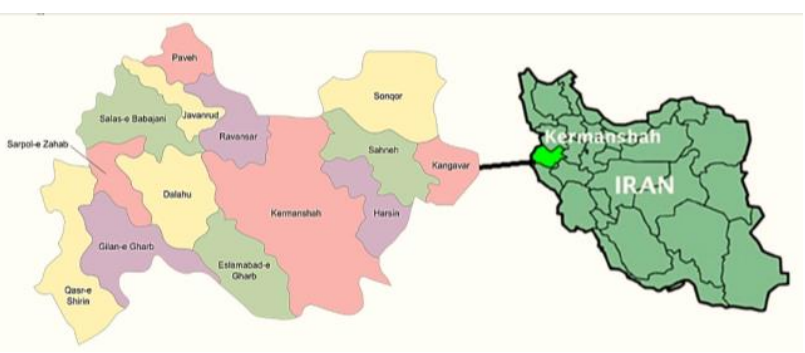

Figure 1. Sarpol-Zahab-Iran

Sarpol-Zahab was one of the two major towns most seriously affected by the 2017 Iran-Iraq earthquake. The historic earthquake of $958 \mathrm{AD}$, with a magnitude of 6.8 , caused the destruction of Sarpol-Zahab and many deaths. The earthquakes and its aftershocks, which continued throughout the first months of the year, affected the groundwater resources in Zagros. The last historical earthquake in this area occurred in 1226 , with a magnitude of 6.5 magnitudes along the high Zagros fault. The magnitude 5.7 earthquake in 1967 and the 5.1 magnitude earthquake in 1983, including the historic and important earthquakes in the city of Sarpol- Zahab, have caused many casualties and losses.

\subsection{Dataset}

The investigated case study is the earthquake that hit SarpolZahab at 18: 18 UTC on 12 November 2017 (Mw 7.3). For the Sarpol-Zahab earthquake, Sentinel-2 and Pleiades-1 images were acquired. The available dataset is made up of one Pleiades-1 satellite optical images (one postseismic), two Sentinel-2 satellite images (one preseismic and one postseismic). Satellite and scene specifications are provided in follow Tables:

\begin{tabular}{|c|c|c|c|c|}
\hline 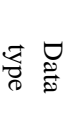 & 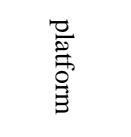 & 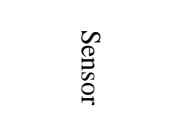 & 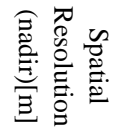 & 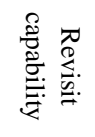 \\
\hline \multirow[t]{2}{*}{ optic } & Pleiades-1 & $\begin{array}{l}\text { Panchromatic } \\
\text { Multispectral }\end{array}$ & $\begin{array}{c}0.5 \\
2\end{array}$ & 1 day \\
\hline & Sentinel-2 & $\begin{array}{l}\text { Panchromatic } \\
\text { Multispectral }\end{array}$ & $\begin{array}{l}10 \\
20\end{array}$ & 5 days \\
\hline
\end{tabular}

Table1.Technical specifications of the sensors used and Specifications of the scenes acquired

\subsection{METHOD}

The main steps of the proposed methodology in this paper (pic1)are the comparison of earthquake-based change maps, prepared using Sentienel-2 data's (before and after the earthquake), with a source map (ground truth) from the high resolution image (after the earthquake only) of the Pleiades-1 satellite. The reason for using this method, examining the justification the use of mid-resolution data (such as Sentinel-2) in generating earthquake-based change maps and measuring the ability of these types of data to detect changes and their effects are included in the subject above.

In this paper, we use one set of data, Sentinel-2 satellite images as candidates, before and after earthquake images.

After Crop and Co-registering the set of images relative to each other and applying the necessary corrections; according to the proposed algorithm (pic1), first, the pair of images (before and after the earthquake) on Sentinel-2 satellite are classified in two common ways of machine learning (SVM) and the neural network (NN), in two classes: "change" and "no change". The reason is the average spatial resolution of these images and the lack of justification pixel-to-pixel study and calculates differencing of the two pre- and post-earthquake images directly. Consequently, by performing this process due to the block-wise or block-to-block checking, the accuracy and validity of the change maps will be improved.

After performing the above steps on two sets of Sentinel-2 images, producing two distinct maps of changes, now we have to validate it with ground truth to measure the accuracy and validity of the produced maps and to check out the effectiveness of these change maps to detect earthquake changes.

In this method, a single Pleiades-1 satellite image related to postearthquake, with the specification inserted in tab1, considered as ground truth.

In the final step, this reference map is compared with the two change maps produced in the previous steps to validate results. Then, a map of the percentage of changes caused by the earthquake in the Sarpol-Zahab city is produced as a process output, which is, in fact, a result of comparison and validation. The general algorithm of the steps is shown in pic2.

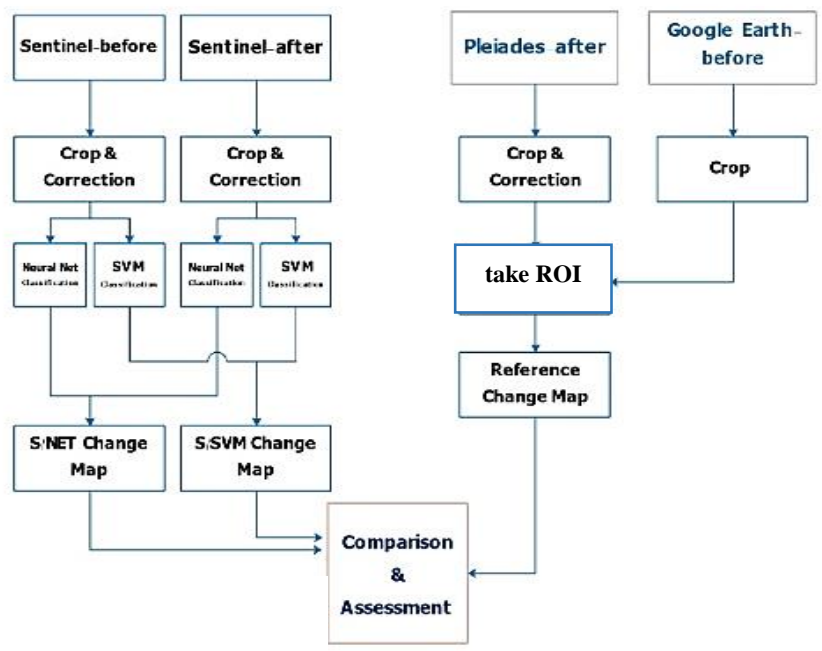

Figure 2. Proposed methodology

Each pixel of the Sentinel image can be considered as representing a building. In the comparison phase, since the images are pixel-to-pixel, so a pixel of the sentinel is compared to a $50 * 50$ pixel dimension.

It should be noted that the use of Google imagery before and after the earthquake, as well as the field reports available during image classification to enhance the accuracy of the classification are considered. [20].

\section{RESULTS}

After classification of the sentinel images (before and after the earthquake, pic2) and preparation the change maps(pic3), Relative to the two methods (SVM classification and the neural network classification) on Sentinel images, so we will have two change maps. 


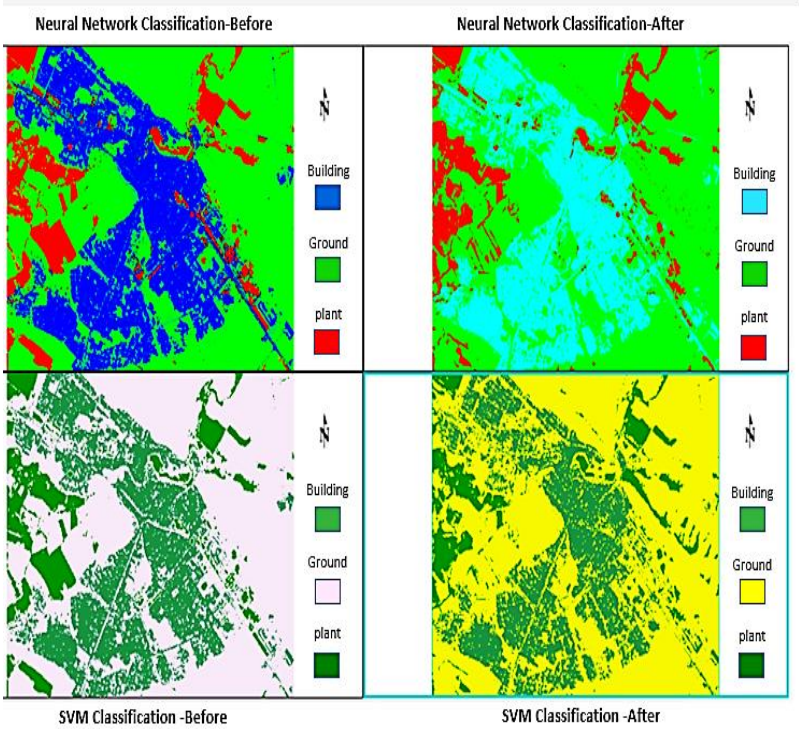

Figure 3. classification of the sentinel images

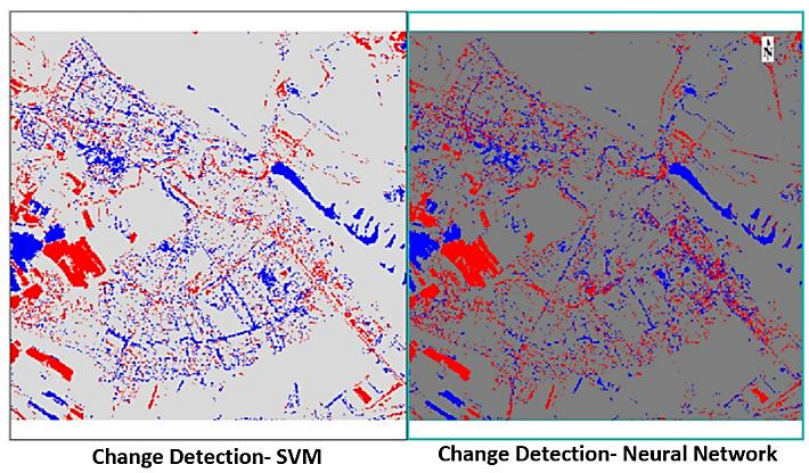

Figure 4. Change Maps

Then, we are going to prepare a reference changes map with ROIs. For this purpose, on a single image Pleiades-1 (after the earthquake), we define ROIs in two categories "change" and "no change" (pic5). Because we work with a single image, we have been using the images and maps available before the earthquake, including Google Earth, for correct and right sampling in the two classes.

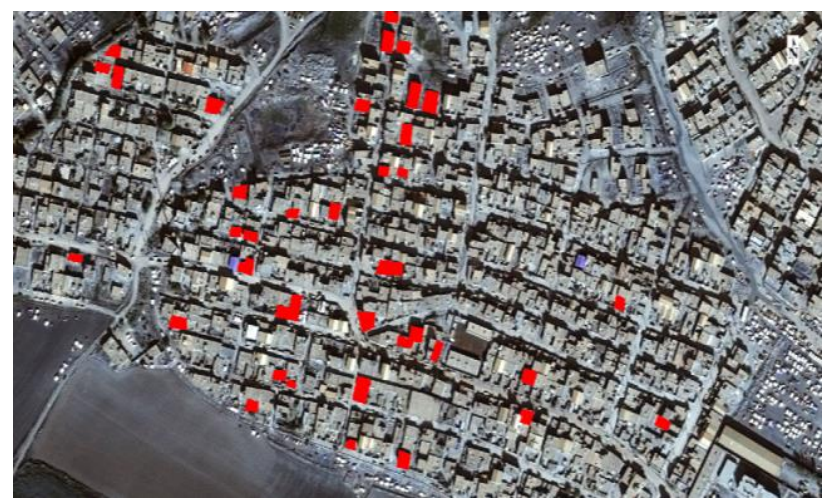

Figure 5. Part of ROIs in category "change"

In the last step, using the confusion matrix, we compared two change map from the Sentinel image to the reference image, and We analyze.

\begin{tabular}{|c|c|c|c|}
\hline \multicolumn{4}{|c|}{ Confusion Matrix for SVM Classification } \\
\hline \multicolumn{4}{|c|}{ Ground Truth (Percent) } \\
\hline Class & CHANGE & $\begin{array}{c}\text { NO } \\
\text { CHANGE }\end{array}$ & OTHERS \\
\hline Change & 86.87 & 20.54 & 13.69 \\
\hline $\begin{array}{c}\text { No } \\
\text { Change }\end{array}$ & 13.13 & 79.46 & 86.31 \\
\hline Total & 100 & 100 & 100 \\
\hline Class & \multicolumn{2}{|c|}{ Prod. Acc. } & User Acc. \\
\hline & \multicolumn{2}{|l|}{ (Percent) } & (Percent) \\
\hline Change & \multicolumn{2}{|l|}{77.22} & 68.10 \\
\hline $\begin{array}{c}\text { No } \\
\text { Change }\end{array}$ & \multicolumn{2}{|l|}{79.46} & 72.16 \\
\hline
\end{tabular}

Table 2. Confusion Matrix for SVM Classification

\begin{tabular}{|c|c|c|c|}
\hline \multicolumn{4}{|c|}{ Confusion Matrix for Neural Network } \\
Classification \\
\hline \multicolumn{4}{|c|}{ Ground Truth (Percent) } \\
\hline Class & CHANGE & $\begin{array}{c}\text { NO } \\
\text { CHANGE }\end{array}$ & OTHERS \\
\hline Change & 81.34 & 16.29 & 14.31 \\
\hline $\begin{array}{c}\text { No } \\
\text { Change }\end{array}$ & 18.66 & 83.71 & 85.69 \\
\hline Total & 100 & 100 & 100 \\
\hline
\end{tabular}

\begin{tabular}{c|cc} 
Class & Prod. Acc. & User Acc. \\
\hline $\begin{array}{c}\text { Change } \\
\text { No }\end{array}$ & (Percent) & (Percent) \\
Change & 69.62 & 67.65 \\
& 75.23 & 70.44
\end{tabular}

Table 3. Confusion Matrix for Neural.Network Classification

\section{CONCLUSIONS}

According to the results, the accuracy of the results in the SVM classification method is better than the Neural Network classification method. Because the accuracy and sensitivity of the SVM classification in the diagnosis of urban complications is better than Neural Network classification.

According to the results of the confusion matrix and the obtained accuracy, it can be seen that medium-resolution images such as Sentinel-2 can also be partially functional and useful in detecting earthquake-related changes. The disadvantages of these types of images in detecting buildings block-wise.

Investigating the distribution of destruction through the maps of changes shows that the destroyed buildings are not a specific pattern. so it can be seen that most of the south and parts of the western part of the Sarkpol Zahab region have the greatest damage. Surveys show that the southern and western parts of the city are along the river and have more densely populated buildings. Another important factor is the fault in this region, which is in the western-southeast. It should be noted that the other 
parts of the city have also been destroyed, but are more dispersed and lacking a specific pattern.

One of the weaknesses of medium resolution images, such as Sentinel, is to detect changes block-to-block and unable to detect individual buildings.

An interesting point in interpreting the results is that all the shelters in the city remain unchanged, which could be helpful in justifying the construction of crisis shelters in each region, and the need to build strategic buildings such as crisis management centers in busy areas.

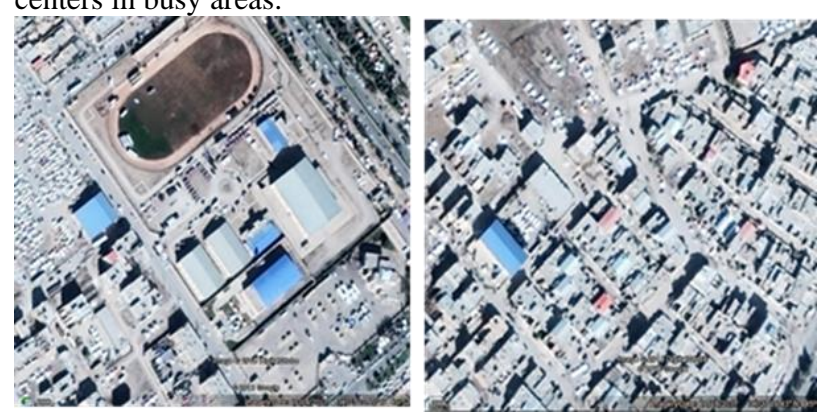

Pic6. An example of the difference in ceiling cover for some buildings that was discovered with ground truths.

\section{REFERENCES}

[1] Bartels, S.A., VanRooyen, M.J., 2011. Medical complications associated with earthquakes. The Lancet 379 (9817), 748-757.

[2] Schweier, C., Markus, M., 2006. Classification of collapsed buildings for fast damage and loss assessment. Bulletin of Earthquake Engineering 4, 177-192.

[3] Voigt, S., Kemper, T., Riedlinger, T., Kiefl, R., Scholte, K., Mehl, H., 2007. Satellite image analysis for disaster and crisismanagement support. IEEE Transactions on Geoscience and Remote Sensing 45 (6), 1520-1528

[4] Sabins, F.F. (1996). Remote Sensing: Principles and Interpretation (3) Freeman, New York.

[5] Huyck, C.K., Mansouri, B., Eguchi, R.T., Houshmand, B., Castner, L.L., and Shinozuka, M. (2002)."Earthquake Damage Detection Algorithm Using Optical and ERS-SAR Satellite Data - Application to the August 17, 1999, Turkey Earthquake." Proceeding of the 7th National US Conference on Earthquake Engineering, Boston.

[6] Saito, K., Spence, R., Going, C. and Markus, M. (2004). "Using High-Resolution Satellite Images for

Post-Earthquake Building Damage Assessment: A Study Following the 26 January 2001 Gujarat

Earthquake.” Earthquake Spectra 20, 145-169.

[7] SINGH, A., 1989, Digital change detection techniques using remotely sensed data. International Journal of Remote Sensing, 10, pp. 989-1003.

[8] Haala, N., and C. Brenner, 1999. Extraction of buildings and trees in urban environments, Photogrammetric Engineering \& Remote Sensing, 54 (1):130-137.

[9] Zhang, Y., 1999. Optimization of building detection in satellite images by combining multi-spectral classification and texture filtering, Journal of Photogrammetry and Remote Sensing, 50:50-60.
[10] Kiema, J.B.K., 2002. Texture analysis and data fusion in the extraction of topographic objects from satellite imagery, International Journal of Remote Sensing, 23(4):767-776.

[11] Lunetta, R.S., and C.D. Elvidge, 1998. Remote Sensing Change Detection: Environmental Monitoring Methods and Applications, Ann Arbor Press, Chelsea, Michigan, USA, 318 p.

[12] Mücher, C.A., K.T. Steinnocher, F.P. Kressler, and C. Heunks, 2000. Land cover characterization and change detection for environmental monitoring of pan-Europe, International Journal of Remote Sensing, 21(6\&7):1159-1181.

[13] Bruzzone, L., and S.B. Serpico, 1997. An iterative technique for detection of land-cover transitions in multi-temporal remote sensing images, IEEE Transactions on Geoscience and Remote Sensing, 35(4):858-867.

[14] Nielsen, A., K. Conradsen, and J. Simpson, 1998. Multivariate alteration detection (MAD) and MAP post processing in multispectral bitemporal image data: new approaches to change detection studies, Remote Sensing of Environment, 64:1-19.

[15] Bouziani, M., Goïta, K., He, D.-C., 2010. Automatic change detection of buildings in the urban environment from very high spatial resolution images using existing geodatabase and prior knowledge. ISPRS Journal of Photogrammetry and Remote Sensing 65, 143-153.

[16] Jensen, J.R., 1983. Urban/Suburban Land Use Analysis, Manual of Remote Sensing. American Society of Photogrammetry, Falls Church, Virginia, pp. 1571-1666.

[17] D.H.A. Al-Khudhairy, I. Caravaggi, and S. Giada,2005. Structural Damage Assessments from Ikonos Data Using Change Detection, Object-Oriented Segmentation, and Classification Techniques, Photogrammetric Engineering \& Remote Sensing Vol. 71, No. 7, July 2005, pp. 825-837.

[18] Richards, J.A. and Jia, X. 2005. Remote sensing digital image analysis. Springer Press. $454 \mathrm{p}$.

[19] Hussain, M., Chen, D., Cheng, A., Wei, H., \& Stanley, D. (2013). Change detection from remotely sensed images: From pixel-based to object-based approaches. ISPRS Journal of Photogrammetry and Remote Sensing,80, 91-106

[20] Chen, G., Hay, G.J., Carvalho, L.M.T., Wulder, M.A., 2012a. Object-based change detection. International Journal of Remote Sensing 33, 4434-4457. 